Journal of Applied Mathematics and Stochastic Analysis, 16:1 (2003), 19-31.

Printed in the USA (C)2003 by North Atlantic Science Publishing Company

\title{
EXISTENCE THEORY FOR SINGLE AND MULTIPLE SOLUTIONS TO SEMIPOSITONE DISCRETE DIRICHLET BOUNDARY VALUE PROBLEMS WITH SINGULAR DEPENDENT NONLINEARITIES
}

\author{
DAQING JIANG and LILI ZHANG \\ Northeast Normal University \\ Department of Mathematics \\ Changchun 130024, PR of China ${ }^{1}$ \\ DONAL O'REGAN \\ National University of Ireland \\ Department of Mathematics \\ Galway, Ireland \\ RAVI P. AGARWAL \\ Florida Institute of Technology \\ Department of Mathematical Sciences \\ Melbourne, FL 32901 USA
}

(Received September, 2002; Revised January, 2003)

In this paper we establish the existence of single and multiple solutions to the semipositone discrete Dirichlet boundary value problem

$$
\left\{\begin{array}{l}
\Delta^{2} y(i-1)+\mu f(i, y(i))=0, \quad i \in\{1,2, \ldots, T\} \\
y(0)=y(T+1)=0
\end{array}\right.
$$

where $\mu>0$ is a constant and our nonlinear term $f(i, u)$ may be singular at $u=0$.

Keywords. Multiple Solutions, Singular Discrete Boundary Value Problem, Semipositone Problem, Upper and Lower Solutions Method.

AMS (MOS) subject classification: 34B15.

\footnotetext{
${ }^{1}$ This work was supported by NNSF of China.
} 


\section{Introduction}

In this paper we establish the existence of single and multiple solutions to the semipositone discrete Dirichlet boundary value problem

$$
\left\{\begin{array}{l}
\Delta^{2} y(i-1)+\mu f(i, y(i))=0, \quad i \in N=\{1,2, \ldots, T\} \\
y(0)=y(T+1)=0
\end{array}\right.
$$

where $\mu>0$ is a constant and $T \in\{1,2, \ldots\}, N^{+}=\{0,1, \ldots, T+1\}$ and $y: N^{+} \rightarrow \mathbf{R}$. Throughout this paper we will assume $f: N \times(0, \infty) \rightarrow \mathbf{R}$ is continuous. As a result our nonlinearity $f(i, u)$ may be singular at $u=0$.

Remark. Recall a map $f: N \times(0, \infty) \rightarrow \mathbf{R}$ is continuous if it is continuous as a map of the topological space $N \times(0, \infty)$ into the topological space $\mathbf{R}$. Throughout this paper the topology on $N$ will be the discrete topology.

We will let $C\left(N^{+}, \mathbf{R}\right)$ denote the class of map $u$ continuous on $N^{+}$(discrete topology), with norm $|u|_{0}=\max _{k \in N^{+}}|u(k)|$. By a solution to $(1.1)$ we mean a $u \in C\left(N^{+}, \mathbf{R}\right)$ such that $u$ satisfies (1.1) for $i \in N$ and $u$ satisfies the boundary (Dirichlet) conditions.

It is of interest to note here that the existence of single and multiple solutions to positone singular boundary value problems in the continuous case have been studied in great detail in the literature $[6,7,8,9,14]$, and only recently (see for example [10, 16]) papers have appeared which discuss the semipositone nonsingular problem. Also recently, R. P. Agarwal and D. O'Regan [5] discussed the semipositone singular problem, and a very general existence theory was presented in [5] which showed, for example, that the boundary value problem

$$
\left\{\begin{array}{l}
y^{\prime \prime}+\mu\left(y^{-\alpha}+y^{\beta}-1\right)=0, \quad 0<t<1 \\
y(0)=y(1)=0, \quad \alpha>0, \quad \beta>1, \quad \mu>0 \text { small }
\end{array}\right.
$$

has a positive solution $y \in C[0,1] \cap C^{2}(0,1)$ with $y(t)>0$ for $t \in(0,1)$. Existence is established in [5] via a general cone fixed point theorem $[6,11]$. However, for the discrete case almost all papers in the literature [2, 4, 12, 13] are devoted to singular positone problems, and only recently in [1] the semipositone nonsingular discrete problem has been discussed.

This paper discusses the existence of single and multiple solutions for semipositone singular discrete problems. Some general existence theorems will be presented in Section 2 and there we will show, for example, that the boundary value problem

$$
\left\{\begin{array}{l}
\Delta^{2} y(i)+\mu\left([y(i)]^{-\alpha}+[y(i)]^{\beta}-1\right)=0, \quad i \in N \\
y(0)=0, \quad y(T+1)=0, \quad \alpha>0, \quad \beta>1, \quad \mu>0 \text { small }
\end{array}\right.
$$

has two nonnegative solutions. Existence in this paper will be established using the upper and lower solutions method in [15] and a general cone fixed point theorem in $[6$, 11].

Theorem 1.1. ${ }^{[6]}$ Let $E=(E,\|\cdot\|)$ be a Banach space and let $K \subset E$ be a cone in $E$, and let $\|\cdot\|$ be increasing with respect to $K$. Also, $r, R$ are constants with $0<r<R$. Suppose $A: \bar{\Omega}_{R} \cap K \rightarrow K\left(\right.$ here $\left.\Omega_{R}=\{x \in E,\|x\|<R\}\right)$ is a continuous, compact map and assume the conditions

$$
x \neq \lambda A(x), \quad \text { for } \lambda \in[0,1) \text { and } x \in \partial \Omega_{r} \cap K
$$


and

$$
\|A x\|>\|x\|, \quad \text { for } x \in \partial \Omega_{R} \cap K
$$

hold. Then $A$ has a fixed point in $K \cap\{x \in E: r \leq\|x\| \leq R\}$.

In this paper we only consider discrete Dirichlet boundary data. It is worth remarking here that we could consider Sturm Liouville boundary data also; however since the arguments are essentially the same (in fact easier if not Dirichlet data) we will leave the details to the reader.

\section{Discrete Equations}

In this section we present some new results for the semipositone singular problem(1.1). Before we prove our main results we first state two well known results [1].

Lemma 2.1. Let $u \in\left(N^{+}, \mathbf{R}\right)$ satisfy $u(i) \geq 0$ for $i \in N^{+}$. If $y \in C\left(N^{+}, \mathbf{R}\right)$ satisfies

$$
\left\{\begin{array}{l}
\Delta^{2} y(i-1)+u(i)=0, \quad i \in N=\{1,2, \ldots, T\} \\
y(0)=y(T+1)=0,
\end{array}\right.
$$

then

$$
y(i) \geq q(i)|y|_{0} \quad \text { for } \quad i \in N^{+}
$$

here

$$
q(i)=\min \left\{\frac{T+1-i}{T+1}, \frac{i}{T}\right\}
$$

Lemma 2.2. Let $\omega$ be the solution of

$$
\left\{\begin{array}{l}
\Delta^{2} y(i-1)+1=0, \quad i \in N \\
y(0)=y(T+1)=0,
\end{array}\right.
$$

then

$$
\omega(i) \leq C_{0} q(i) \text { for } i \in N^{+}
$$

here

$$
C_{0}=\frac{(T+1)^{2}}{2} \text { and } q(i)=\min \left\{\frac{T+1-i}{T+1}, \frac{i}{T}\right\}
$$

Remark. In fact $\omega(i)=\frac{i(T+1-i)}{2}, i \in N^{+}$.

Theorem 2.1. $\quad$ Suppose the following condition are satisfied:

$$
\begin{gathered}
\left\{\begin{array}{l}
f: N \times(0, \infty) \rightarrow \mathbf{R} \text { is continuous and there exists } \\
\text { a constant } M>0 \text { with } f(i, u)+M \geq 0 \\
\text { for all } i \in N \text { and } u \in(0, \infty) ;
\end{array}\right. \\
\left\{\begin{array}{c}
f^{*}(i, u)=f(i, u)+M \leq g(u)+h(u) \text { for }(i, u) \in N \times(0, \infty) \\
\text { with } g>0 \text { continuous and nonincreasing on }(0, \infty), h \geq 0 \\
\text { continuous on }[0, \infty) \text { and } \frac{h}{g} \text { nondecreasing on }(0, \infty) ;
\end{array}\right. \\
\exists K_{0} \geq 0 \text { with } g(a b) \leq K_{0} g(a) g(b) \quad \forall a>0, b>0 ;
\end{gathered}
$$


and

$$
\left\{\begin{array}{l}
\text { there exists contants } L>M \text { and } \epsilon_{0}>0 \text { such that } g(u)>L \\
\text { for } u \in\left(0, \epsilon_{0}\right), \text { and } f^{*}(i, u)>L \text { for all }(i, u) \in N \times\left(0, \epsilon_{0}\right) .
\end{array}\right.
$$

Then (1.1) has a solution $y \in C\left(N^{+}, \mathbf{R}\right)$ with $y(i)>0$ for $i \in N,|y+\phi|_{0}<r$, here $\phi(i)=\mu M \omega(i)$ ( $\omega$ is as in Lemma 2.2).

Proof. Choose $\delta>0$ and $\delta<r$ with

$$
\frac{1}{g\left(1-\frac{\mu M C_{0}}{r}\right)\left\{1+\frac{h(r)}{g(r)}\right\}} \int_{\delta}^{r} \frac{d u}{g(u)}>\frac{1}{2} \mu K_{0} T(T+1) .
$$

Let $m_{0} \in\{1,2, \ldots\}$ be chosen so that $\frac{1}{m_{0}}<\min \left\{\frac{\delta}{2}, \epsilon_{0}\right\}$ and let $N_{0}=\left\{m_{0}, m_{0}+1, \ldots\right\}$.

To show (1.1) has a nonnegative solution $y \in C\left(N^{+}, \mathbf{R}\right)$ with $|y+\phi|_{0}<r$, we will show

$$
\left\{\begin{array}{l}
\Delta^{2} y(i-1)+\mu f^{*}(i, y(i)-\phi(i))=0, \quad i \in N \\
y(0)=y(1)=0
\end{array}\right.
$$

has a solution $y_{1} \in C\left(N^{+}, \mathbf{R}\right)$ with $y_{1}(i)>\phi(i)$ for $i \in N$ and $\left|y_{1}\right|_{0}<r$, here $f^{*}(i, u)$ is as in (2.2).

If this is true then $u(i)=y_{1}(i)-\phi(i)\left(i \in N^{+}\right)$is a nonnegative solution (positive on $N$ ) of (1.1), and $|u+\phi|_{0}<r$, since

$$
\begin{aligned}
& \Delta^{2} u(i-1)=\Delta^{2} y_{1}(i-1)-\Delta^{2} \phi(i-1) \\
& =-\mu f^{*}\left(i, y_{1}(i)-\phi(i)\right)+\mu M \\
& =-\mu\left(f\left(i, y_{1}(i)-\phi(i)\right)+M\right)+\mu M \\
& =-\mu f\left(i, y_{1}(i)-\phi(i)\right) \\
& =-\mu f(i, u(i)), \quad i \in N .
\end{aligned}
$$

As a result we will concentrate our study on (2.7).

The idea is to first show that

$$
\left\{\begin{array}{l}
\Delta^{2} y(i-1)+\mu f^{*}(i, y(i)-\phi(i))=0, \quad i \in N \\
y(0)=\frac{1}{m}, \quad y(T+1)=\frac{1}{m}, \quad m \in N_{0}
\end{array}\right.
$$

has a solution $y_{m}$ for each $m \in N_{0}$ with $y_{m}(i) \geq \frac{1}{m}$ and $y_{m}(i) \geq \phi(i)$ for $i \in N^{+}$, and $\left|y_{m}\right|_{0}<r$.

We have the following claim.

Claim $1 \alpha_{m}(i)=\frac{1}{m}+l w(i)+\phi(i)=\frac{1}{m}+(l+\mu M) w(i), i \in N^{+}$is a (strict) lower solution for problem $(2.8)^{m}$, here $0<l<\min \left\{\mu(L-M), \frac{\varepsilon_{0}-\frac{1}{m_{0}}}{|w|_{0}}\right\}, m \in N_{0}$.

Proof. Notice $\alpha_{m}(0)=\alpha_{m}(T+1)=\frac{1}{m}$, and

$$
\begin{aligned}
& \Delta^{2} \alpha_{m}(i-1)+\mu f_{m}^{*}\left(i, \alpha_{m}(i)-\phi(i)\right) \\
= & (l+\mu M) \Delta^{2} w(i)+\mu f^{*}\left(i, \frac{1}{m}+l w(i)\right) \\
= & -(l+\mu M)+\mu f^{*}\left(i, \frac{1}{m}+l w(i)\right) \\
\geq & -(l+\mu M)+\mu L \\
> & 0, \quad i \in N,
\end{aligned}
$$

since $l w(i)+\frac{1}{m} \leq l|w|_{0}+\frac{1}{m_{0}}<\varepsilon_{0}$, and $l<\mu(L-M)$.

In order to seek upper solutions of $(2.8)^{m}$, we consider the following problem

$$
\left\{\begin{array}{l}
\Delta^{2} y(i-1)+\mu g(y(i)-\phi(i))\left(1+\frac{h(r)}{g(r)}\right)=0, \quad i \in N, \\
y(0)=\frac{1}{m}, \quad y(T+1)=\frac{1}{m}, \quad m \in N_{0} .
\end{array}\right.
$$


In the same way as Claim 1, we can easily prove $\alpha_{m}(i)=\frac{1}{m}+l w(i)+\phi(i)=$ $\frac{1}{m}+(l+\mu M) w(i)$ is also a (strict) lower solution of $(2.9)^{m}$.

Let $\beta_{m}^{0} \in C\left(N^{+}, \mathbf{R}\right)$ be the unique solution of the discrete boundary value problem

$$
\left\{\begin{array}{l}
\Delta^{2} y(i-1)+\mu g\left(\alpha_{m}(i)-\phi(i)\right)\left(1+\frac{h(r)}{g(r)}\right)=0, \quad i \in N, \\
y(0)=\frac{1}{m}, \quad y(T+1)=\frac{1}{m}, \quad m \in N_{0} .
\end{array}\right.
$$

Then we have

$$
\beta_{m}^{0}(i)=\frac{1}{m}+\mu \sum_{j=1}^{T} G(i, j) g\left(\alpha_{m}(j)-\phi(j)\right)\left(1+\frac{h(r)}{g(r)}\right), \quad i \in N^{+} ;
$$

here $G(i, j)$ is the Green's function to the problem

$$
\left\{\begin{array}{l}
-\Delta^{2} u(i-1)=0, \quad i \in N \\
u(0)=u(T+1)=0
\end{array}\right.
$$

so

$$
G(i, j)= \begin{cases}\frac{j(T+1-i)}{T+1}, & 0 \leq j \leq i-1 \\ \frac{i(T+1-j)}{T+1}, & i \leq j \leq T+1\end{cases}
$$

Then we have

$$
\begin{aligned}
\beta_{m}^{0}(i) & =\frac{1}{m}+\mu \sum_{j=1}^{T} G(i, j) g\left(\frac{1}{m}+l w(j)\right)\left(1+\frac{h(r)}{g(r)}\right) \\
& \geq \frac{1}{m}+\mu\left(1+\frac{h(r)}{g(r)}\right) g\left(\frac{1}{m_{0}}+l|w|_{0}\right) \sum_{j=1}^{T} G(i, j) \\
& =\frac{1}{m}+\mu\left(1+\frac{h(r)}{g(r)}\right) g\left(\frac{1}{m_{0}}+l|w|_{0}\right) w(i) \\
& \geq \frac{1}{m}+\mu\left(1+\frac{h(r)}{g(r)}\right) L w(i) \\
& \geq \frac{1}{m}+\mu L w(i) \\
& \geq \frac{1}{m}+(l+\mu M) w(i) \\
& =\alpha_{m}(i), \quad i \in N^{+} .
\end{aligned}
$$

Thus we obtain

$$
\begin{aligned}
& \Delta^{2} \beta_{m}^{0}(i-1)+\mu g\left(\beta_{m}^{0}(i)-\phi(i)\right)\left(1+\frac{h(r)}{g(r)}\right) \\
= & \mu\left(1+\frac{h(r)}{g(r)}\right)\left[g\left(\beta_{m}^{0}(i)-\phi(i)\right)-g\left(\alpha_{m}(i)-\phi(i)\right)\right] \leq 0, \quad i \in N,
\end{aligned}
$$

so that $\beta_{m}^{0}$ is an upper solution for problem $(2.9)^{m}$.

If we now take $\alpha_{m}^{0} \equiv \alpha_{m}$, we have that $\alpha_{m}^{0}$ and $\beta_{m}^{0}$ are, respectively, a lower and an upper solution of $(2.9)^{m}$ with $\alpha_{m}^{0}(i) \leq \beta_{m}^{0}(i)$, for all $i \in N^{+}$. So by the upper and lower solutions method in [15], we know that there exists a solution $\beta_{m} \in C\left(N^{+}, \mathbf{R}\right)$ of $(2.9)^{m}$ such that

$$
\alpha_{m}(i)=\alpha_{m}^{0}(i) \leq \beta_{m}(i) \leq \beta_{m}^{0}(i), \quad \forall i \in N^{+} .
$$

Now we claim that $\left|\beta_{m}\right|_{0}<r$. Suppose this is false, i.e., suppose $\left|\beta_{m}\right|_{0} \geq r$. Since $\Delta^{2} \beta_{m}(i-1) \leq 0$ on $N$ and $\beta_{m}(0)-\frac{1}{m}=\beta_{m}(T+1)-\frac{1}{m}=0$, then $\beta_{m}(i)-\frac{1}{m} \geq$ $q(i)\left|\beta_{m}-\frac{1}{m}\right|_{0}, \quad i \in N^{+}$(from Lemma 2.1). Thus, $\beta_{m}(i) \geq \frac{1}{m}+q(i)\left(\left|\beta_{m}\right|_{0}-\frac{1}{m}\right) \geq$ $q(i)\left|\beta_{m}\right|_{0} \geq q(i) r, \quad i \in N^{+}$. Also, there exists $\sigma_{m} \in N$ with $\Delta \beta_{m}(i) \geq 0$ on $\left[0, \sigma_{m}\right)=$ $\left\{0, \ldots, \sigma_{m}-1\right\}, \quad \Delta \beta_{m}(i) \leq 0$ on $\left[\sigma_{m}, T+1\right)=\left\{\sigma_{m}, \ldots \ldots, T\right\}$ (note $\Delta^{2} \beta_{m}(i-1) \leq 0$ on $N)$, and $\beta_{m}\left(\sigma_{m}\right)=\left|\beta_{m}\right|_{0} \geq r$. Notice also for $i \in N^{+}$that

$$
\beta_{m}(i)-\phi(i)=\beta_{m}(i)\left[1-\frac{\mu M w(i)}{\beta_{m}(i)}\right] \geq \beta_{m}(i)\left[1-\frac{\mu M C_{0}}{r}\right],
$$


since $\beta_{m}(i) \geq q(i)\left|\beta_{m}\right|_{0} \geq q(i) r$, and $w(i) \leq C_{0} q(i)$ for $i \in N^{+}$. Thus

$$
\beta_{m}(i)-\phi(i) \geq \beta_{m}(i)\left[1-\frac{\mu M C_{0}}{r}\right]>0, \quad \text { for } \quad i \in N,
$$

since $r>\mu M C_{0}$. Thus for $i \in N$, we have

$$
-\Delta^{2} \beta_{m}(i-1)=\mu g\left(\beta_{m}(i)-\phi(i)\right)\left\{1+\frac{h(r)}{g(r)}\right\}
$$

and this together with (2.11) yields

$$
-\Delta^{2} \beta_{m}(i-1) \leq \mu K_{0} g\left(1-\frac{\mu M C_{0}}{r}\right)\left\{1+\frac{h(r)}{g(r)}\right\} g\left(\beta_{m}(i)\right), \quad i \in N .
$$

Sum the inequality $(2.12)$ from $j\left(1 \leq j<\sigma_{m}\right)$ to $\sigma_{m}$ to obtain

$$
\Delta \beta_{m}(j-1) \leq \Delta \beta_{m}\left(\sigma_{m}\right)+\mu K_{0} g\left(1-\frac{\mu M C_{0}}{r}\right)\left\{1+\frac{h(r)}{g(r)}\right\} \sum_{s=j}^{\sigma_{m}} g\left(\beta_{m}(s)\right),
$$

i.e.,

$$
\Delta \beta_{m}(j) \leq \Delta \beta_{m}\left(\sigma_{m}\right)+\mu K_{0} g\left(1-\frac{\mu M C_{0}}{r}\right)\left\{1+\frac{h(r)}{g(r)}\right\} \sum_{s=j}^{\sigma_{m-1}} g\left(\beta_{m}(s+1)\right) .
$$

Since $\Delta \beta_{m}\left(\sigma_{m}\right) \leq 0$, and $\beta_{m}(s+1) \geq \beta_{m}(j+1)$ when $j \leq s<\sigma_{m}$, then we have

$$
\Delta \beta_{m}(j) \leq g\left(\beta_{m}(j+1)\right) \mu K_{0} g\left(1-\frac{\mu M C_{0}}{r}\right)\left\{1+\frac{h(r)}{g(r)}\right\}\left(\sigma_{m}-j\right), \quad j<\sigma_{m},
$$

i.e.,

$$
\frac{\Delta \beta_{m}(j)}{g\left(\beta_{m}(j+1)\right)} \leq \mu K_{0} g\left(1-\frac{\mu M C_{0}}{r}\right)\left\{1+\frac{h(r)}{g(r)}\right\}\left(\sigma_{m}-j\right), \quad j<\sigma_{m} .
$$

Since $g\left(\beta_{m}(j+1)\right) \leq g(u) \leq g\left(\beta_{m}(j)\right)$ for $\beta_{m}(j) \leq u \leq \beta_{m}(j+1)$, when $j<\sigma_{m}$, then we have

$$
\int_{u(j)}^{u(j+1)} \frac{d u}{g(u)} \leq \frac{\Delta \beta_{m}(j)}{g\left(\beta_{m}(j+1)\right)} \leq \mu K_{0} g\left(1-\frac{\mu M C_{0}}{r}\right)\left\{1+\frac{h(r)}{g(r)}\right\}\left(\sigma_{m}-j\right), \quad j<\sigma_{m},
$$

and then sum the above from 0 to $\sigma_{m}-1$ to obtain

$$
\int_{\frac{1}{m}}^{\beta_{m}\left(\sigma_{m}\right)} \frac{d u}{g(u)} \leq \mu K_{0} g\left(1-\frac{\mu M C_{0}}{r}\right)\left\{1+\frac{h(r)}{g(r)}\right\} \frac{\sigma_{m}\left(\sigma_{m}+1\right)}{2} .
$$

Similarly, sum the inequality (2.12) from $\sigma_{m}$ to $j\left(\sigma_{m}<j<T+1\right)$ to obtain

$$
-\Delta \beta_{m}(j) \leq-\Delta \beta_{m}\left(\sigma_{m}-1\right)+\mu K_{0} g\left(1-\frac{\mu M C_{0}}{r}\right)\left\{1+\frac{h(r)}{g(r)}\right\} \sum_{s=\sigma_{m}}^{j} g\left(\beta_{m}(s)\right), \quad j>\sigma_{m} .
$$

Since $\Delta \beta_{m}\left(\sigma_{m}-1\right) \geq 0, \beta_{m}(s) \geq \beta_{m}(j)$ for $\sigma_{m} \leq s \leq j$, then we have

$$
-\Delta \beta_{m}(j) \leq \mu K_{0} g\left(1-\frac{\mu M C_{0}}{r}\right)\left\{1+\frac{h(r)}{g(r)}\right\}\left(j-\sigma_{m}+1\right), \quad j>\sigma_{m} .
$$


So we have

$$
\int_{\beta_{m}(j+1)}^{\beta_{m}(j)} \frac{d u}{g(u)} \leq \frac{-\Delta \beta_{m}(j)}{g\left(\beta_{m}(j)\right)} \leq \mu K_{0} g\left(1-\frac{\mu M C_{0}}{r}\right)\left\{1+\frac{h(r)}{g(r)}\right\}\left(j-\sigma_{m}+1\right), \quad j>\sigma_{m},
$$

and then we sum the above from $\sigma_{m}$ to $T$ to obtain

$$
\int_{\frac{1}{m}}^{\beta_{m}\left(\sigma_{m}\right)} \frac{d u}{g(u)} \leq \mu K_{0} g\left(1-\frac{\mu M C_{0}}{r}\right)\left\{1+\frac{h(r)}{g(r)}\right\} \frac{\left(T-\sigma_{m}+1\right)\left(T-\sigma_{m}+2\right)}{2} .
$$

Now, (2.13) and (2.14) imply

$$
\int_{\delta}^{r} \frac{d u}{g(u)} \leq \int_{\frac{1}{m}}^{\beta_{m}\left(\sigma_{m}\right)} \frac{d u}{g(u)} \leq \frac{1}{2} \mu K_{0} g\left(1-\frac{\mu M C_{0}}{r}\right)\left\{1+\frac{h(r)}{g(r)}\right\} T(T+1) .
$$

This contradicts (2.6) and consequently $\left|\beta_{m}\right|_{0}<r$.

Observe that

$$
\begin{aligned}
f^{*}\left(i, \beta_{m}(i)-\phi(i)\right) & =g\left(\beta_{m}(i)-\phi(i)\right)\left(1+\frac{h\left(\beta_{m}(i)-\phi(i)\right)}{g\left(\beta_{m}(i)-\phi(i)\right)}\right) \\
& \leq g\left(\beta_{m}(i)-\phi(i)\right)\left\{1+\frac{h(r)}{g(r)}\right\}, \quad i \in N .
\end{aligned}
$$

Thus we have

$$
\begin{aligned}
& \Delta^{2} \beta_{m}(i-1)+\mu f^{*}\left(i, \beta_{m}(i)-\phi(i)\right) \\
= & -\mu g\left(\beta_{m}(i)-\phi(i)\right)\left\{1+\frac{h(r)}{g(r)}\right\}+\mu f^{*}\left(i, \beta_{m}(i)-\phi(i)\right) \\
\leq & 0, \quad i \in N,
\end{aligned}
$$

so that $\beta_{m}$ is an upper solution for $(2.8)^{m}$. This together with Claim 1, implies that $\alpha_{m}$ and $\beta_{m}$ are, respectively, a lower and an upper solution for problem $(2.8)^{m}$ with $\alpha_{m}(i) \leq \beta_{m}(i)$, for all $i \in N^{+}$. So we conclude that $(2.8)^{m}$ has a solution $y_{m} \in C\left(N^{+}, \mathbf{R}\right)$ such that

$$
\alpha_{m}(i) \leq y_{m}(i) \leq \beta_{m}(i), \quad i \in N^{+} .
$$

Thus we have

$$
\left|y_{m}\right|_{0}<r, \quad y_{m}(i)-\phi(i) \geq \frac{1}{m}+l w(i)>l w(i), \quad i \in N^{+} .
$$

The Arzela-Ascoli Theorem guarantees the existence of a subsequence $N_{1}$ of $N_{0}$ and a function $y \in C\left(N^{+}, \mathbf{R}\right)$ with $y_{m} \rightarrow y$ in $C\left(N^{+}, \mathbf{R}\right)$ as $m \rightarrow \infty$ through $N_{1}$. Also $y(0)=y(T+1)=0$ and

$$
|y|_{0}<r, \quad y(i)-\phi(i) \geq l w(i), \quad i \in N^{+}
$$

Now fix $i \in N$. Then $y_{m}, m \in N_{1}$, satisfies

$$
y_{m}(i)=\frac{1}{m}+\sum_{j=1}^{T} G(i, j) f^{*}\left(j, y_{m}(j)-\phi(j)\right) .
$$

Also

$$
0<l \min _{k \in N} w(k) \leq y_{m}(j)<r, \text { for } j \in N \text { and } m \in N_{1} \text {. }
$$


Let $m \rightarrow \infty$ (through $\left.N_{1}\right)$ in (2.16) to obtain

$$
y(i)=\sum_{j=1}^{T} G(i, j) f^{*}(j, y(j)-\phi(j))
$$

We can do this for each $i \in N$ and so

$$
\Delta^{2} y(i-1)+f^{*}(i, y(i)-\phi(i))=0, \quad i \in N .
$$

Theorem 2.2. Suppose (2.1)-(2.4) hold. In addition assume the following hold:

$$
\left\{\begin{array}{l}
f^{*}(i, u)=f(i, u)+M \geq g_{1}(u)+h_{1}(u) \text { with } \\
g_{1}>0 \text { continuous and nonincreasing on }(0, \infty), h_{1} \geq 0 \\
\text { continuous on }[0, \infty) \text { and } \frac{h_{1}}{g_{1}} \text { nondecreasing on }(0, \infty)
\end{array}\right.
$$

and

$$
\left\{\begin{array}{l}
\exists R>r \text { with } \\
\frac{R g_{1}\left(\frac{\epsilon}{T+1} R\right)}{g_{1}(R) g_{1}\left(\frac{\epsilon}{T+1} R\right)+g_{1}(R) h_{1}\left(\frac{\epsilon}{T+1} R\right)} \leq \mu \sum_{j=1}^{T} G(\sigma, j) ;
\end{array}\right.
$$

here $\epsilon>0$ is any constant (choose and fix it) so that $1-\frac{\mu M C_{0}}{R} \geq \epsilon$ (note $\epsilon$ exists since $R>r>\mu M C_{0}$ ) and $G(i, j)$ is the Green's function for

$$
\left\{\begin{array}{l}
-\Delta^{2} y(i-1)=0, \quad i \in N \\
y(0)=y(T+1)=0
\end{array}\right.
$$

and $\sigma \in N^{+}$is such that

$$
\sum_{j=1}^{T} G(\sigma, j)=\max _{i \in N^{+}} \sum_{j=1}^{T} G(i, j) .
$$

Then (1.1) has a solution $y \in C\left(N^{+}, \mathbf{R}\right)$ with $y(i)>0$ for $i \in N, r<|y+\phi|_{0} \leq R$, here $\phi(i)=\mu M \omega(i)(\omega$ is as in Lemma 2.2).

Proof. Choose $\delta>0$ and $\delta<r$ with

$$
\frac{1}{g\left(1-\frac{\mu M C_{0}}{r}\right)\left\{1+\frac{h(r)}{g(r)}\right\}} \int_{\delta}^{r} \frac{d u}{g(u)}>\frac{1}{2} \mu K_{0} T(T+1) .
$$

Let $m_{0} \in\{1,2, \ldots\}$ be chosen so that $\frac{1}{m_{0}}<\frac{\delta}{2}, \frac{1}{m_{0}}<\frac{\epsilon R}{T+1}, \frac{1}{m_{0}}<\frac{1}{T+1}\left(r-\mu M C_{0}\right)$, and $N_{0}=$ $\left\{m_{0}, m_{0}+1, \ldots\right\}$.

To show (1.1) has a nonnegative solution $y \in C\left(N^{+}, \mathbf{R}\right)$ with $r<|y+\phi|_{0} \leq R$, we will show

$$
\left\{\begin{array}{l}
\Delta^{2} y(i-1)+\mu f^{*}(i, y(i)-\phi(i))=0, \quad i \in N \\
y(0)=y(1)=0
\end{array}\right.
$$

has a solution $y_{2} \in C\left(N^{+}, \mathbf{R}\right)$ with $y_{2}(i)>\phi(i)$ for $i \in N$ and $r<\left|y_{2}\right|_{0} \leq R$, here $f^{*}(i, u)$ is as in (2.2). If this is true, then $u(i)=y_{2}(i)-\phi(i)$ is a nonnegative solution (positive on $N$ ) of (1.1). As a result we will concentrate our study on (2.20).

The idea is to first show that

$$
\left\{\begin{array}{l}
\Delta^{2} y(i-1)+\mu f_{m}(i, y(i)-\phi(i))=0, \quad i \in N \\
y(0)=\frac{1}{m}, \quad y(T+1)=\frac{1}{m}, \quad m \in N_{0}
\end{array}\right.
$$


has a solution $y_{m}$ for each $m \in N_{0}$ with $y_{m}(i) \geq \frac{1}{m}$ and $y_{m}(i) \geq \phi(i)$ for $i \in N^{+}$, and $r<\left|y_{m}\right|_{0} \leq R$, here

$$
f_{m}(i, u)=\left\{\begin{array}{l}
f^{*}(i, u), \quad u \geq \frac{1}{m} \\
f^{*}\left(i, \frac{1}{m}\right), \quad u \leq \frac{1}{m}
\end{array}\right.
$$

Note $f_{m}(i, u) \geq 0$ for $u \in(-\infty, \infty)$.

Fix $m \in N_{0}$. Let $E=\left(C\left(N^{+}, \mathbf{R}\right),|\bullet|_{0}\right)$ and

$$
K=\left\{u \in C\left(N^{+}, \mathbf{R}\right): u(k) \geq q(k)|u|_{0} \text { for } k \in N^{+}\right\},
$$

here $q(k)$ is as in Lemma 2.1.

Let $A: K \rightarrow C\left(N^{+}, \mathbf{R}\right)$ be defined by

$$
(A y)(i)=\frac{1}{m}+\mu \sum_{j=1}^{T} G(i, j) f_{m}(j, y(j)-\phi(j)), \quad i \in N^{+} .
$$

A standard argument [3] implies $A: K \rightarrow C\left(N^{+}, \mathbf{R}\right)$ is continuous and completely continuous. Next we show $A: K \rightarrow K$. If $u \in K$, then clearly $(A u)(i) \geq 0$ for $i \in N^{+}$. Also notice

$$
\left\{\begin{array}{l}
\Delta^{2}(A u)(i) \leq 0 \quad \text { on } N \\
(A u)(0)=(A u)(T+1)=\frac{1}{m}
\end{array}\right.
$$

then by Lemma 2.1, $(A u)(i)-\frac{1}{m} \geq q(i)\left|A u-\frac{1}{m}\right|_{0}$ for $i \in N^{+}$, and so we have $(A u)(i) \geq$ $\frac{1}{m}+q(i)\left(|A u|_{0}-\frac{1}{m}\right) \geq q(i)|A u|_{0}$. Consequently $A u \in K$ so $A: K \rightarrow K$. Let

$$
\Omega_{1}=\left\{u \in C\left(N^{+}, \mathbf{R}\right):\left|u_{0}\right|<r\right\} \text { and } \Omega_{2}=\left\{u \in C\left(N^{+}, \mathbf{R}\right):\left|u_{0}\right|<R\right\}
$$

We first show

$$
y \neq \lambda A y \text { for } \lambda \in[0,1) \text { and } y \in K \cap \partial \Omega_{1} .
$$

Suppose this is false i.e. suppose there exists $y \in K \cap \partial \Omega_{1}$ and $\lambda \in[0,1)$ with $y=$ $\lambda A y$. We can assume $\lambda \neq 0$. Now since $y=\lambda A y$ we have

$$
\left\{\begin{array}{l}
\Delta^{2} y(i-1)+\lambda \mu q(t) f_{m}(i, y(i)-\phi(i))=0, \quad i \in N, \\
y(0)=y(T+1)=\frac{\lambda}{m}
\end{array}\right.
$$

with $y(i) \geq q(i) r$ for $i \in N^{+}$. Since $\Delta^{2} y(i-1) \leq 0$ on $N$ and $y \geq \frac{\lambda}{m}$ on $N^{+}$, there exists $i_{0} \in N$ with $\Delta y(i) \geq 0$ on $\left[0, i_{0}\right)=\left\{0, \ldots, i_{0}-1\right\}, \Delta y(i) \leq 0$ on $\left[i_{0}, T+1\right)=\left\{i_{0}, \ldots . ., T\right\}$ and $y\left(i_{0}\right)=|y|_{0}=r$. Notice also for $i \in N^{+}$that

$$
y(i)-\phi(i)=y(i)\left[1-\frac{\mu M w(i)}{y(i)}\right] \geq y(i)\left[1-\frac{\mu M C_{0}}{r}\right],
$$

since $y(i) \geq q(i) r$ and $w(i) \leq C_{0} q(i)$ for $i \in N^{+}$. Thus

$$
y(i)-\phi(i)=y(i)\left[1-\frac{\mu M C_{0}}{r}\right]>0 \quad \text { for } \quad i \in N,
$$

since $r>\mu M C_{0}$. Also notice

$$
\begin{aligned}
y(i)-\phi(i) & \geq y(i)\left[1-\frac{\mu M C_{0}}{r}\right] \\
& \geq q(i) r\left[1-\frac{\mu M C_{0}}{r}\right] \\
& \geq \frac{1}{T+1} r\left[1-\frac{\mu M C_{0}}{r}\right] \\
& \geq \frac{1}{m_{0}}, \quad i \in N .
\end{aligned}
$$


Thus we have

$$
f_{m}(y(i)-\phi(i))=f^{*}(y(i)-\phi(i)) \leq g(y(i)-\phi(i))+h(y(i)-\phi(i)), \quad i \in N .
$$

Now for $i \in N$ we have

$$
-\Delta^{2} y(i-1) \leq \mu g(y(i)-\phi(i))\left\{1+\frac{h(y(i)-\phi(i))}{g(y(i)-\phi(i))}\right\}
$$

and this together with (2.23) yields

$$
-\Delta^{2} y(i-1) \leq \mu K_{0} g\left(1-\frac{\mu M C_{0}}{r}\right)\left\{1+\frac{h(r)}{g(r)}\right\} g(y(i)), \quad i \in N .
$$

The reasoning used to obtain (2.13) and (2.14) in Theorem 2.1, yields

$$
\int_{\frac{\lambda}{m}}^{y\left(i_{0}\right)} \frac{d u}{g(u)} \leq \mu K_{0} g\left(1-\frac{\mu M C_{0}}{r}\right)\left\{1+\frac{h(r)}{g(r)}\right\} \frac{i_{0}\left(i_{0}+1\right)}{2},
$$

and

$$
\int_{\frac{\lambda}{m}}^{y\left(i_{0}\right)} \frac{d u}{g(u)} \leq \mu K_{0} g\left(1-\frac{\mu M C_{0}}{r}\right)\left\{1+\frac{h(r)}{g(r)}\right\} \frac{\left(T-i_{0}+1\right)\left(T-i_{0}+2\right)}{2} .
$$

Now (2.25) and (2.26) imply

$$
\int_{\delta}^{r} \frac{d u}{g(u)}=\int_{\delta}^{y\left(i_{0}\right)} \frac{d u}{g(u)} \leq \frac{1}{2} \mu K_{0} g\left(1-\frac{\mu M C_{0}}{r}\right)\left\{1+\frac{h(r)}{g(r)}\right\} T(T+1) .
$$

This contradicts (2.19) and consequently (2.22) is true.

Next we show

$$
|A y|_{0} \geq|y|_{0} \text { for } y \in K \cap \partial \Omega_{2} .
$$

To see this let $y \in K \cap \partial \Omega_{2}$ so $y(i) \geq q(i) R$ for $i \in N^{+}$. Also for $i \in N^{+}$we have

$$
y(i)-\phi(i) \geq y(i)-\mu M C_{0} q(i) \geq y(i)\left[1-\frac{\mu M C_{0}}{R}\right] \geq \epsilon y(i) \geq \epsilon q(i) R .
$$

Thus for $i \in N$ we have

$$
y(i)-\phi(i) \geq \epsilon q(i) R \geq \frac{\epsilon}{T+1} R
$$

and so

$$
f_{m}(y(i)-\phi(i))=f^{*}(y(i)-\phi(i)) \geq g_{1}(y(i)-\phi(i))+h_{1}(y(i)-\phi(i)), \quad i \in N,
$$

since $y(i)-\phi(i) \geq \frac{\epsilon}{T+1} R>\frac{1}{m_{0}}$ for $i \in N$. As a result we have

$$
\begin{aligned}
(A y)(\sigma) & =\frac{1}{m}+\mu \Sigma_{j=1} T G(\sigma, j) f_{m}(y(j)-\phi(j)) \\
& \geq \mu \sum_{j=1}^{T} G(\sigma, j) g_{1}(y(j)-\phi(j))\left\{1+\frac{h_{1}(y(j)-\phi(j))}{g(y(i)-\phi(i))}\right\} \\
& \geq \mu g_{1}(R)\left\{1+\frac{h_{1}\left(\frac{\epsilon}{T+1} R\right)}{g_{1}\left(\frac{\epsilon}{T+1} R\right)}\right\} \sum_{j=1}^{T} G(\sigma, j) \\
& \geq R=|y|_{0},
\end{aligned}
$$


using(2.18). Thus $|A y|_{0} \geq|y|_{0}$, so (2.27) is true.

Now Theorem 1.1 implies $A$ has a fixed point $y_{m} \in K \bigcap\left(\bar{\Omega}_{2} \backslash \Omega_{1}\right)$ i.e., $r \leq\left|y_{m}\right|_{0} \leq R$ and $y_{m}(i) \geq r q(i)$ for $i \in N^{+}$In fact $\left|y_{m}\right|_{0}>r$ (note if $\left|y_{m}\right|_{0}=r$ then essentially the same argument from (2.24)-(2.26) will yield a contradiction). In addition $y_{m}(i)>\phi(i)$ for $i \in N$ since

$$
y_{m}(i) \geq r q(i)>\mu M C_{0} q(i) \geq \mu M w(i)=\phi(i) .
$$

Thus $y_{m}$ is a solution of $(2.21)^{m}$.

Essentially the same argument as in the proof of Theorem 2.1, guarantees the existence of a subsequence $N_{1}$ of $N_{0}$ and a function $y \in C\left(N^{+}, \mathbf{R}\right)$ with $y_{m} \rightarrow y$ in $C\left(N^{+}, \mathbf{R}\right)$ as $m \rightarrow \infty$ through $N_{1}$. Also, $y \in C\left(N^{+}, \mathbf{R}\right)$ is a nonnegative solution of $(2.20)$ such that $r \leq|y|_{0} \leq R, y(i) \geq q(i) r$ for $i \in N^{+}$. In addition $y(i)>\phi(i)$ for $i \in N$. Finally it is easy to see that $|y|_{0}>r$ (note if $\left|y_{0}\right|=r$ then essentially the argument from (2.24)-(2.26) will yield a contradiction).

Theorem 2.3. Suppose (2.1)-(2.5) and (2.17)-(2.18) hold. Then (1.1) has two solutions $y_{n} \in C\left(N^{+}, \mathbf{R}\right)(n=1,2)$ with $y_{n}(i)>0$ for $i \in N, 0<\left|y_{1}+\phi\right|_{0}<r<$ $\left|y_{2}+\phi\right|_{0} \leq R$, here $\phi(i)=\mu M w(i)$ ( $w$ is as in Lemma 2.2).

Proof. The existence of $y_{1}$ follows from Theorem 2.1 and the existence of $y_{2}$ follows from Theorem 2.2.

Example. Consider the boundary value problem

$$
\left\{\begin{array}{l}
\Delta^{2} y(i)+\mu\left([y(i)]^{-\alpha}+[y(i)]^{\beta}-1\right)=0, \quad i \in N \\
y(0)=0, \quad y(T+1)=0, \quad \alpha>0, \quad \beta>1
\end{array}\right.
$$

with $\mu \in\left(0, \mu_{0}\right)$ is such that

$$
\left[\mu_{0} T(T+1)(\alpha+1)\right]^{\frac{1}{\alpha}}+\frac{\mu_{0}(T+1)^{2}}{2} \leq 1 .
$$

Then (2.28) have two solutions $y_{n}$ with $y_{n}(i)>0$, for $i \in N, \quad n=1,2$, and $0<$ $\left|y_{1}+\phi\right|_{0}<1<\left|y_{2}+\phi\right|_{0}$, here $\phi(i)=\mu w(i)=\frac{\mu i(T+1-i)}{2}, \quad i \in N^{+}$.

To see this we will apply Theorem 2.3 with (here $R>1$ will be chosen below; in fact here we choose $R$ so that $\epsilon=\frac{1}{2}$ works i.e., we choose $\mathrm{R}$ so that $1-\frac{\mu M C_{0}}{R} \geq \frac{1}{2}$ )

$$
M=1, w(i)=\frac{i(T+1-i)}{2}, \phi(i)=\frac{\mu i(T+1-i)}{2},
$$

and

$$
g(y)=g_{1}(y)=y^{-\alpha}, h(y)=h_{1}(y)=y^{\beta}, \epsilon=\frac{1}{2}, K_{0}=1, C_{0}=\frac{(T+1)^{2}}{2} .
$$

Clearly $(2.1),(2.2),(2.3),(2.4),(2.5),(2.17)$ hold. Also note

$$
\frac{1}{g\left(1-\frac{\mu M C_{0}}{r}\right)\left\{1+\frac{h(r)}{g(r)}\right\}} \int_{0}^{r} \frac{d u}{g(u)}=\left(1-\frac{\mu(T+1)^{2}}{2 r}\right)^{\alpha} \frac{1}{1+r^{\alpha+\beta}} \frac{r^{\alpha+1}}{\alpha+1} .
$$

Now (2.6) holds with $r=1$ since

$$
\begin{aligned}
& \frac{1}{2} \mu K_{0} T(T+1)=\frac{\mu T(T+1)}{2}<\frac{\mu_{0} T(T+1)}{2} \leq \frac{\left(1-\frac{\mu_{0}(T+1)^{2}}{2}\right)^{\alpha}}{2(\alpha+1)} \\
& \leq \frac{\left(1-\frac{\mu(T+1)^{2}}{2}\right)^{\alpha}}{2(\alpha+1)}=\frac{1}{g\left(1-\frac{\mu M C_{0}}{r}\right)\left\{1+\frac{h(r)}{g(r)}\right\}} \int_{0}^{r} \frac{d u}{g(u)}
\end{aligned}
$$


from (2.29). Finally notice (2.18) is satisfied for R large since

$$
\frac{R g_{1}\left(\frac{\epsilon}{T+1} R\right)}{g_{1}(R) g_{1}\left(\frac{\epsilon}{T+1} R\right)+g_{1}(R) h_{1}\left(\frac{\epsilon}{T+1} R\right)}=\frac{R^{1+\alpha}}{1+\left(\frac{\epsilon}{T+1}\right)^{\alpha+\beta} R^{\alpha+\beta}} \rightarrow 0
$$

as $R \rightarrow \infty$, since $\beta>1$. Thus all the conditions of Theorem 2.3 are satisfied so existence of two positive solutions is guaranteed.

\section{References}

[1] Agarwal, R.P. and O'Regan, D., Nonpositive discrete boundary value problems, Nonlinear Analysis, 39(2000), 207-215.

[2] Agarwal, R.P. and O'Regan, D., Singular discrete boundary value problems, Appl. Math. Letters, 12(1999), 127-131.

[3] Agarwal, R.P. and O'Regan, D., Boundary value problems for discrete equations, Appl. Math. Letters, 10(1997), 83-89.

[4] Agarwal, R.P. and O'Regan, D., Singular discrete $(n, p)$ boundary value problems, Applied Math. Letters 12(1999), 113-119.

[5] Agarwal, R.P. and O'Regan, D., Semipositone Dirichlet boundary value problem with singular dependent nonlinearities, Houston. J. Math., to appear.

[6] Agarwal, R.P. and O'Regan, D., Existence theorem for single and multiple solutions to singular positone boundary value problems, Jour. Differential Equations, 175(2001), 393-414.

[7] Agarwal, R.P. and O'Regan, D., Twin solutions to singular Dirichlet problems, J. Math. Anal. Appl. 240(1999), 433-445.

[8] Agarwal, R.P. and O'Regan, D., Twin solutions to singular boundary value problems, Proc. Amer. Math. Soc. 128(7)(2000),2085-2094.

[9] Agarwal, R.P. and O'Regan, D., Multiplicity results for singular conjugate, focal, and $(N, P)$ problems, Jour. Differential Equations 170(2001), 142-156.

[10] Bobisud, L.E., Behavior of solutions for a Robin problem, Jour. Differential Equations, 85(1990), 91-104.

[11] Deimling, K., Nonlinear Functional Analysis, Springer Verlag, Berlin 1985.

[12] Henderson, J., Singular boundary value problems for difference equations, Dynamics Systems and Applications, 271-282(1992).

[13] Henderson, J., Singular boundary value problems for higher order difference equations, Proceedings of the First World Congress on Nonlinear Analysis, (Edited by V. Lakshmikantham), pp. 1139-1150, Walter de Gruyter, 1994.

[14] Jiang, D.Q., Multiple positive solutions to singular boundary value problems for superlinear higher-order ODEs, Computers and Mathematics with Applications 40(2000),249-259. 
[15] Jiang, D.Q., Pang, P.Y.H. and Agarwal, R.P., Upper and lower solutions method and a superlinear singular discrete boundary value problem, Dynamics Systems and Applications, to appear.

[16] Mengseng, L., On a fourth order eigenvalue problem, Advances in Math., 29(2000), 91-93. 


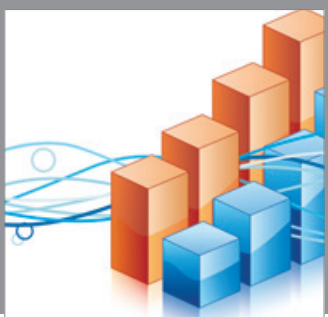

Advances in

Operations Research

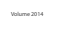

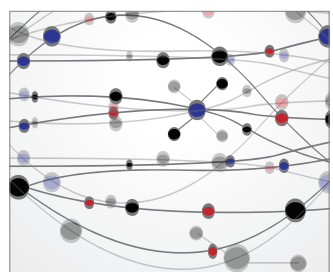

\section{The Scientific} World Journal
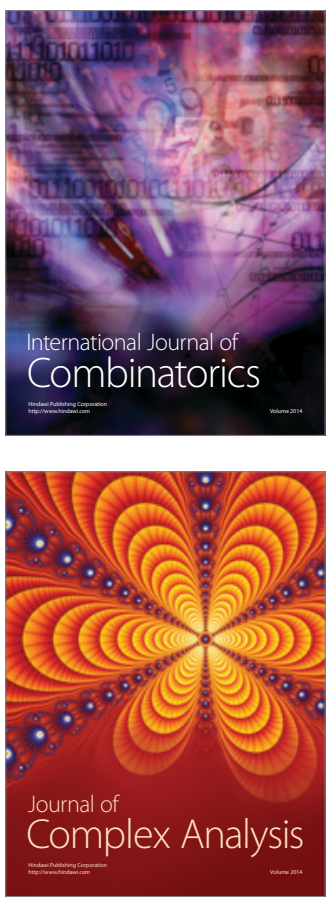

International Journal of

Mathematics and

Mathematical

Sciences
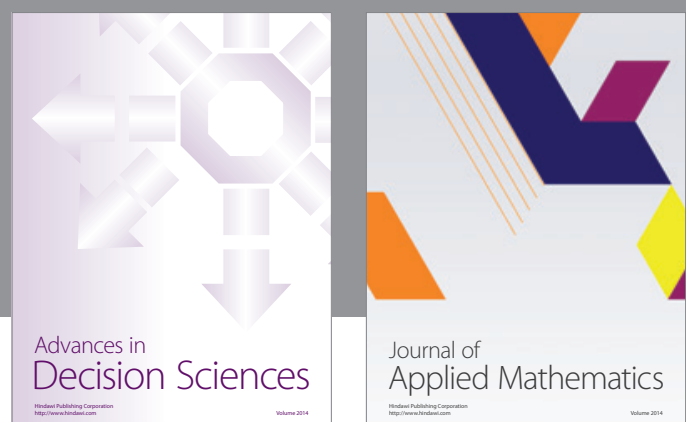

Journal of

Applied Mathematics
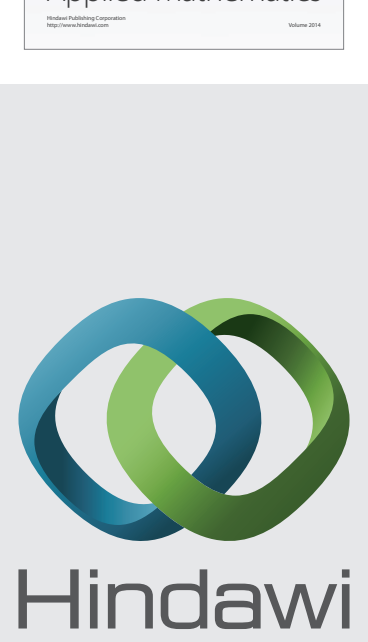

Submit your manuscripts at http://www.hindawi.com
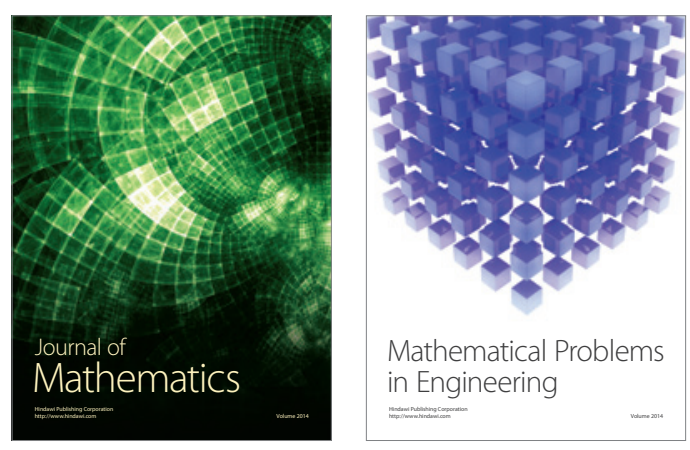

Mathematical Problems in Engineering
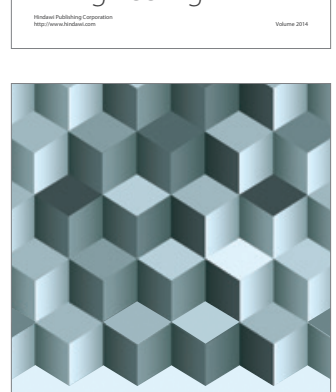

Journal of

Function Spaces
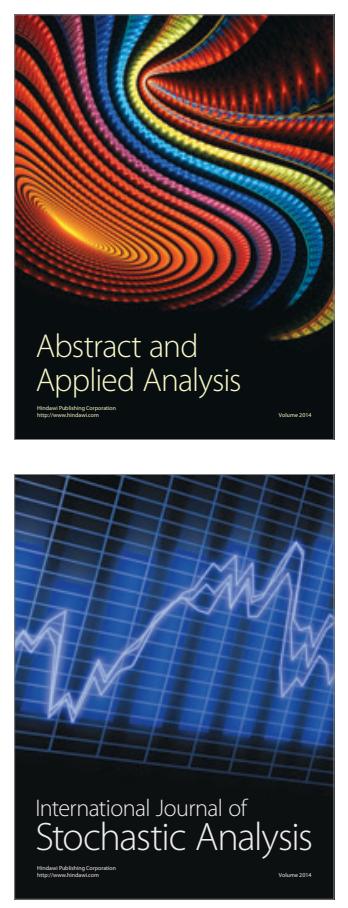

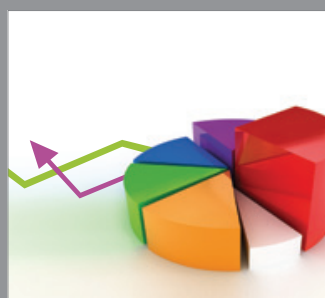

ournal of

Probability and Statistics

Promensencen
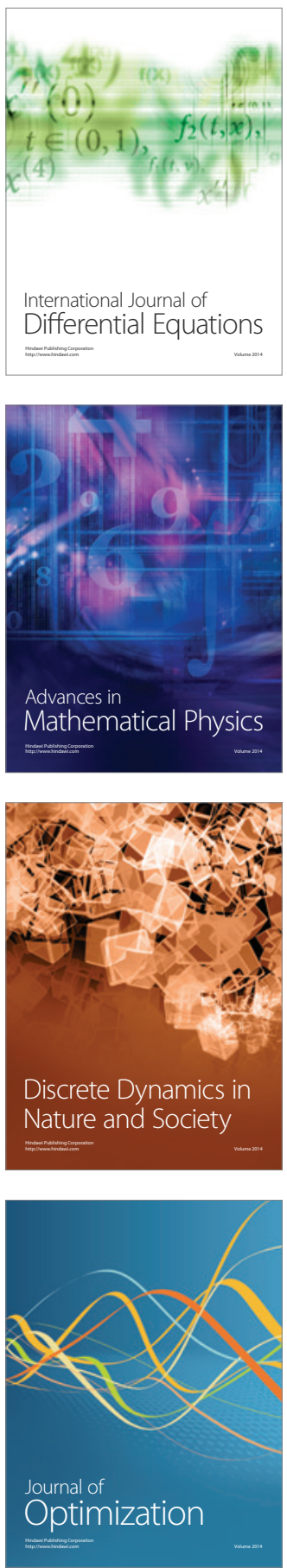